\title{
Testing the Vainshtein Mechanism using Stars and Galaxies
}

\author{
Jeremy Sakstein \\ Institute of Cosmology and Gravitation, University of Portsmouth, Portsmouth PO1 3FX, UK \\ jeremy.sakstein@port.ac.uk \\ Kazuya Koyama \\ Institute of Cosmology and Gravitation, University of Portsmouth, Portsmouth PO1 3FX, UK \\ kazuya.koyama@port.ac.uk
}

\begin{abstract}
The Vainshtein mechanism is of paramount importance in many alternative theories of gravity. It hides deviations from general relativity in the solar system whilst allowing them to drive the acceleration of the cosmic expansion. Recently, a class of theories have emerged where the mechanism is broken inside astrophysical objects. In this essay we look for novel probes of these theories by deriving the modified properties of stars and galaxies. We show that main-sequence stars are colder, less luminous and more ephemeral than general relativity predicts. Furthermore, the circular velocities of objects orbiting inside galaxies are slower and the lensing of light is weaker. We discuss the prospects for testing these theories using the novel phenomena presented here in light of current astrophysical surveys.
\end{abstract}

\section{Introduction}

Many alternative theories of gravity attempt to explain the acceleration of the cosmic expansion by adding additional scalar degrees of freedom to the general relativity (GR) action. These invariably make predictions that violate solar system tests of GR and require non-linear screening mechanisms ${ }^{1}$ to remain viable. Screening mechanisms decouple cosmological and solar system scales such that GR is satisfied locally but the modifications are active in determining the cosmology. The Vainshtein mechanism ${ }^{2}$ is incredibly successful at this and is exhibited in a number of well-studied alternatives to GR! ${ }^{[-9}$ To illustrate the mechanism, we will use the quartic galileon! 10

$\mathcal{L}=-\frac{M_{\mathrm{pl}}^{2}}{2} \partial_{\mu} \phi \partial^{\mu} \phi-\frac{M_{\mathrm{pl}}^{2}}{\Lambda^{4}} X\left[(\square \phi)^{2}-\phi_{\mu \nu} \phi^{\mu \nu}\right]-\left(\phi^{\mu} \phi^{\nu} \phi_{\mu \nu} \square \phi-\phi^{\mu} \phi_{\mu \nu} \phi_{\rho} \phi^{\rho \nu}\right)+\alpha \phi T$,

where $T$ is the trace of the energy-momentum tensor and $X=-1 / 2 g^{\mu \nu} \partial_{\mu} \phi \partial_{\nu} \phi$. This describes a scalar field on a fixed Minkowski background and is not a fully covariant theory. The action contains higher-order derivatives but the equations of motion (EOM) are second-order due to the presence of the galileon symmetry $\phi \rightarrow$ $\phi+b_{\mu} x^{\mu}+c_{\mu}$ and the theory is therefore free of the Ostrogradski instability ${ }^{[1]}$ The final term represents a coupling of the scalar to matter and results in an additional 
or fifth-force $F_{5}=-\alpha \nabla \phi$ (per unit mass). The field sourced by a non-relativistic density distribution $\rho$ is governed by the generalised Poisson equation

$$
\frac{1}{r^{2}} \frac{\mathrm{d}}{\mathrm{d} r}\left[r^{2} \phi^{\prime}+\frac{2}{\Lambda^{4}} \phi^{\prime 3}\right]=8 \pi \alpha G \rho,
$$

which can be integrated once to give an algebraic relation for the ratio of the fifthto Newtonian-force:

$$
\frac{F_{\mathrm{N}}}{F_{5}}+\left(\frac{r_{\mathrm{V}}}{r}\right)^{2}\left(\frac{F_{5}}{F_{\mathrm{N}}}\right)^{3}=2 \alpha^{2},
$$

where $r_{\mathrm{V}}=\sqrt{2} G M / \alpha \Lambda^{2}$ is the Vainshtein radius. When $r \gg r_{\mathrm{V}}$ the first term dominates and the fifth-force is a factor of $2 \alpha^{2}$ larger than the Newtonian force. This is the unscreened regime. When $r \ll r_{\mathrm{V}}$ this enhancement is suppressed by a factor of $\left(r / r_{\mathrm{V}}\right)^{2}$ and the force is screened. This is shown schematically in figure 1. This mechanism is extremely efficient: the Vainshtein radius of the Sun is of $\mathcal{O}(\mathrm{pc}){ }^{[12}$ which precludes the possibility of astrophysical effects.

When one tries to promote (1) to a fully covariant theory of gravity i.e. to make gravity dynamical, the second-order nature of the EOM is destroyed due to couplings of the scalar to curvature tensors that vanish on a Minkowski background. One way to resolve this is to add additional couplings to curvature tensors that conspire to leave the EOM second-order. In this way, one is led to the Horndeski class of theories, $\sqrt[7]{13}$ the most general scalar-tensor theory whose EOM are second-order. The Vainshtein mechanism works perfectly here but large subsets of the theory are in tension with cosmological probes ${ }^{14}[15$ Recently, it has been realised that there are theories beyond Horndeski $16 \sqrt{20}$ where the EOM are second-order due to the presence of hidden constraints. The direct covariantisation of (1) falls into this class of theories and is hence viable. In this theory of gravity, the Vainshtein mechanism works perfectly outside objects ${ }^{21}$ but inside it is broken. If the metric sourced by a spherically symmetric body is

$$
\mathrm{d} s^{2}=-(1+2 \Phi) \mathrm{d} t^{2}+(1-2 \Psi) \delta_{i j} \mathrm{~d} x^{i} \mathrm{~d} x^{j}
$$

the metric potentials satisfy 2122

$$
\begin{aligned}
& \frac{\mathrm{d} \Phi}{\mathrm{d} r}=\frac{G_{\mathrm{N}} M(r)}{r^{2}}+\frac{\Upsilon}{4} G_{\mathrm{N}} M^{\prime \prime}(r) \\
& \frac{\mathrm{d} \Psi}{\mathrm{d} r}=\frac{G_{\mathrm{N}} M(r)}{r^{2}}-\frac{5 \Upsilon}{4} \frac{G_{\mathrm{N}} M^{\prime}(r)}{r} .
\end{aligned}
$$

The final terms are modifications to the GR equations whose strength is controlled by the parameter $\Upsilon \equiv\left(\dot{\phi}_{0} / \Lambda\right)^{4}$, where $\phi_{0}$ is the cosmological value of the scalar.

The density of most astrophysical objects decreases outwards so the second term in (5) is negative and acts to weaken the strength of gravity. The aim of this essay is to use (5) and (6) to derive the modified properties of stars and galaxies in order to look for new observational signatures. 


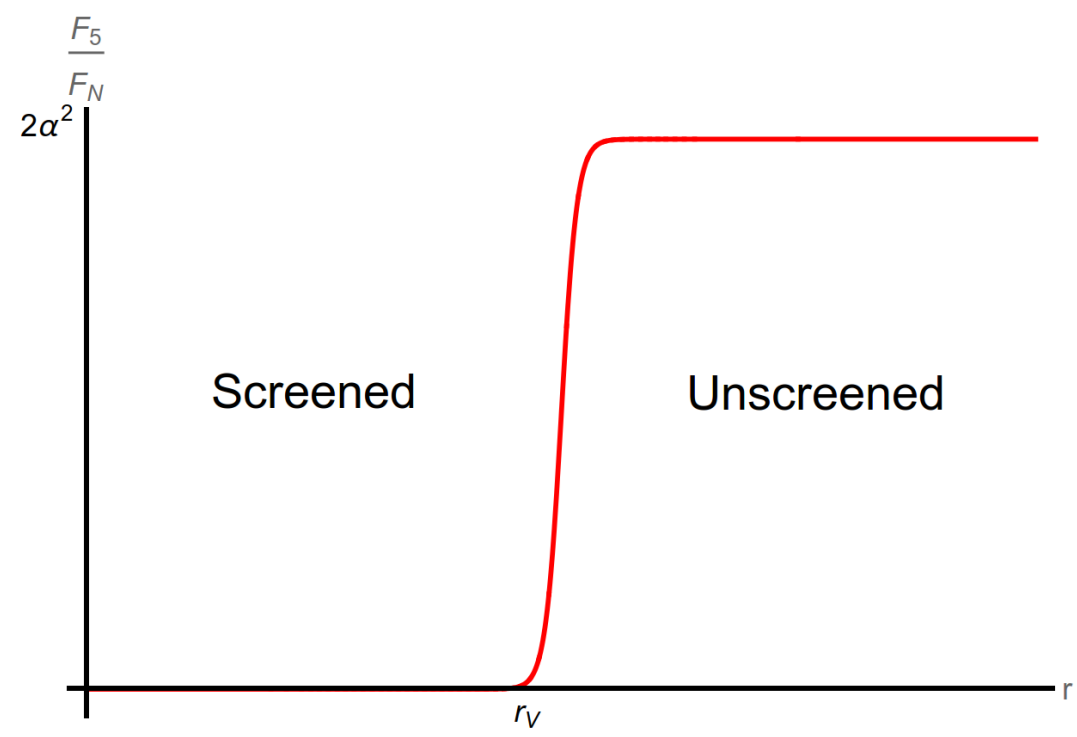

Fig. 1. Vainshtein screening of a point source located at the origin.

\section{Stellar Structure Tests}

Gravity's influence on the structure of non-relativistic stars is completely encoded in the hydrostatic equilibrium equation

$$
\frac{1}{\rho} \frac{\mathrm{d} P}{\mathrm{~d} r}=-\frac{\mathrm{d} \Phi}{\mathrm{d} r}
$$

which, using equation (5), is

$$
\frac{\mathrm{d} P}{\mathrm{~d} r}=-\frac{G_{\mathrm{N}} M(r) \rho(r)}{r^{2}}-\frac{\Upsilon}{4} G_{\mathrm{N}} \rho(r) M^{\prime \prime}(r) .
$$

Solving equation (8) is a difficult problem since one must simultaneously solve for other processes such as nuclear burning and convection. In order to isolate the effects of modified gravity, it is instructive to make the Eddington approximation, which assumes that the entropy gradient $\left(\propto T^{3} / \rho\right)$ is constant. This decouples the hydrostatic equilibrium equation from the non-gravitational equations. With this approximation, one can solve the hydrostatic equilibrium equation numerically to find the ratio of the stellar luminosity to the GR prediction ( $\mathrm{sec}^{21}$ for the technical details). This is shown for a solar mass star as a function of $\Upsilon$ in figure 2(a) and as a function of mass for various values of $\Upsilon$ in figure 2(b). One can see that stronger modifications result in stars that are less luminous than their GR counterparts. This is because the reduced gravity requires a lower nuclear burning rate to maintain equilibrium and less energy is released per unit time as a result. The deviation from GR at low masses is larger because the stellar luminosity is more sensitive to the strength of gravity in lower mass stars. ${ }^{23}$ 


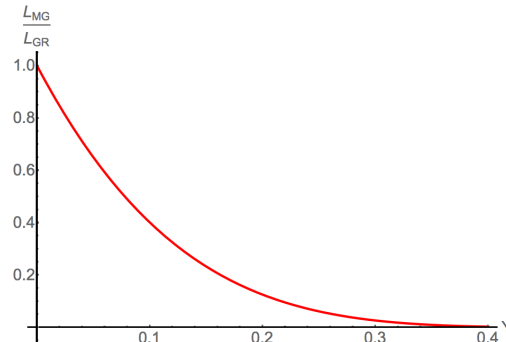

(a)

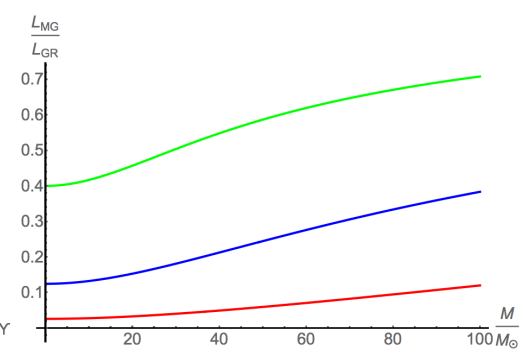

(b)

Fig. 2. The ratio of the stellar luminosity to the GR prediction as a function of $\Upsilon$ for a solar mass star 2(a) and the same ratio as a function of stellar mass for $\Upsilon=0.3$ (red), $\Upsilon=0.2$ (blue) and $\Upsilon=0.1$ (green) 2(b) .

In order to move beyond these approximations and make predictions that can be compared with data we have modified the stellar structure code MESA $24 \sqrt[25]{25}$ to include the modified hydrostatic equilibrium equation (8). MESA includes a consistent treatment of all stellar processes neglected above. In figures 3(a) and 3(b) we show the Hertzprung-Russell diagram for $M_{\odot}$ and $2 M_{\odot}$ stars with metallicity $Z=0.02$ (solar metallicity) for various values of $\Upsilon$. One can immediately see the predictions of the previous analysis are borne out in the main-sequence: stars of fixed mass and metallicity are dimmer than their GR counterparts. Furthermore, one can see that their effective temperatures are lower. Plotted also in $3(\mathrm{a})$ are the points where the stars have the same age as our Sun. One can see that stars evolve at a slower rate when the modifications are stronger. The modifications to the post-main-sequence are negligible and all tracks converge to the GR prediction. The tracks for GR stars with $Z=0.03$ are also shown. One can see that they are nearly coincident with the tracks for $\Upsilon=0.1$ on the main-sequence and so the effects of modified gravity are degenerate with changing the stellar metallicity. Metal-enriched stars have higher opacities and are less luminous than metal-poor stars. The two tracks can be distinguished in the post-main-sequence phase since the modified gravity track returns to the GR prediction for the same metallicity whereas the $Z=0.03$ track remains at lower luminosities (at fixed temperature). This deviation in the main- but not post-main-sequence serves as a potential probe of these theories: it predicts a non-agreement between the fitting of the age, distance and metallicity of globular clusters using the main-sequence and red giant branch independently.

\section{Galaxy Tests}

The rotation curves of galaxies are sensitive to the metric potential $\Phi$ because this determines the circular velocity of non-relativistic objects via the relation

$$
\frac{v^{2}}{r}=\frac{\mathrm{d} \Phi}{\mathrm{d} r} .
$$




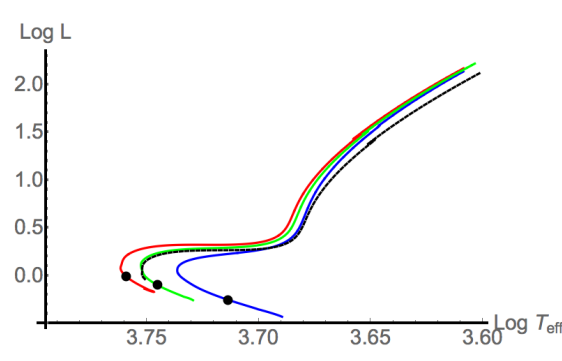

(a)

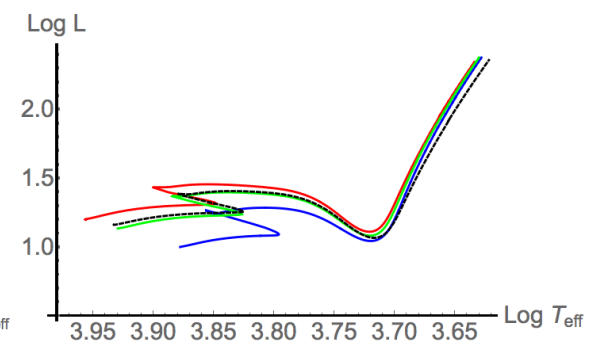

(b)

Fig. 3. The Hertzprung-Russell diagram for $M_{\odot} 3(\mathrm{a})$ and $2 M_{\odot} 3(\mathrm{~b})$ stars. All stars have metallicity $Z=0.02$ except for the black dashed tracks, which correspond to GR stars with $Z=0.03$. The red tracks show the GR prediction and the blue and green tracks show the evolution of the same star when $\Upsilon=0.3$ and $\Upsilon=0.1$ respectively. The filled black circles in $3(\mathrm{a})$ indicate the point at which the age of the star is equal to the solar age, $4.6 \times 10^{9} \mathrm{yr}$.

The most precise observations are made in the Milky Way and so we concentrate on Milky Way-like galaxies, which are well described by the Nevarro-Frenk-White density profile ${ }^{26}$

$$
\rho(r)=\frac{\rho_{\mathrm{s}}}{\frac{r}{r_{\mathrm{s}}}\left(1+\frac{r}{r_{\mathrm{s}}}\right)^{2}} .
$$

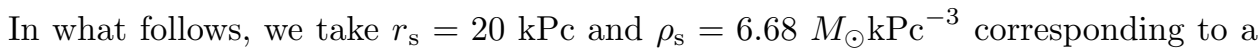
Milky Way-like halo of mass $\left.10^{12} M_{\odot} \cdot 27\right] 28$ Using equation (5) with this profile for various values of $\Upsilon$ we find the rotation curves plotted in figure 4(a), The rising portion of the curve at small radii is measured using stellar kinematics and one can see that deviations from GR are negligible here. The flat portion of the curve, measured using $\mathrm{H} \alpha$ or $21 \mathrm{~cm}$ emission, lies increasingly below the GR prediction when the modifications are stronger due to the weakening of the gravitational force. This difference can be up to $20 \mathrm{~km} / \mathrm{s}$ for $\Upsilon \sim \mathcal{O}(1)$. There is then a clear signature of the theory: a fit to the NFW profile using stellar kinematics should predict larger circular velocities than are observed separately using $\mathrm{H} \alpha$ or $21 \mathrm{~cm}$ measurements. Current observational data is precise enough to allow fitting to several alternative models and, indeed, rotation curves have already provided strong constraints on other alternative theories of gravity $[29 \sqrt{32}$

The motion of light is governed by the lensing potential $\Phi+\Psi$. Integrating equations (5) and (6), we plot the ratio of the lensing potential to $\Phi$ for the same Milky Way-like galaxy in figure 4(b) for various values of $\Upsilon$. One can see that the ratio increasingly lies below the GR prediction of unity when the modifications are stronger. This means that the lensing mass of individual galaxies (mass inferred using lensing) is less than their dynamical mass (mass inferred using the motion of non-relativistic satellites). Figure 4(b) indicates that the deviations from GR can be up to $10 \%$ and lie in the strong lensing regime. Strong lensing is a particularly good probe of modified gravity. Indeed ${ }^{34}$ have already used strong lensing to constrain 


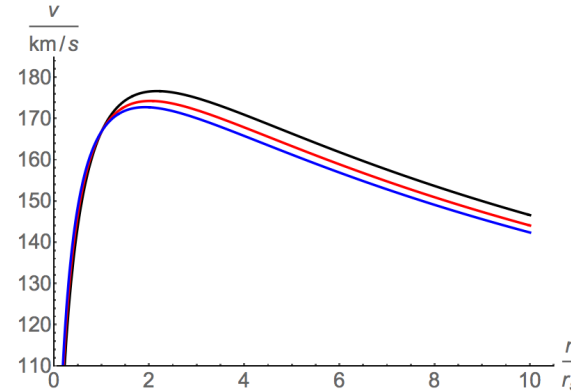

(a)

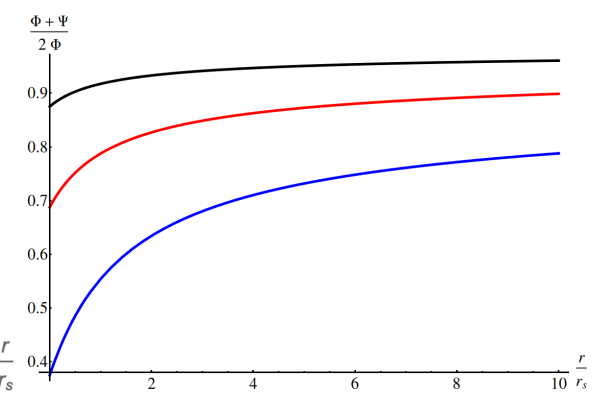

(b)

Fig. 4. The rotation curves 4(a) and lensing potential 4(b) for a Milky Way-like NFW halo with $r_{\mathrm{s}}=20 \mathrm{kPc}$ and $\rho_{\mathrm{s}}=6.68$. In figure $4(\mathrm{a})$ the black, red, and blue curves correspond to GR, $\Upsilon=0.3$ and $\Upsilon=0.5$ respectively. In figure $4(\mathrm{~b})$ the modified gravity predictions with $\Upsilon=0.1$, $\Upsilon=0.3$, and $\Upsilon=0.5$ are plotted using black, red and blue curves respectively, the GR prediction is unity.

deviations of the parameter $\gamma$, defined by

$$
\mathrm{d} s^{2}=-\left(1+2 \frac{G_{\mathrm{N}} M}{r}\right) \mathrm{d} t^{2}+\left(1-2 \gamma \frac{G M}{r}\right) \mathrm{d} x^{2},
$$

from unity to the $5 \%$ level. Their constraint cannot be used to constrain our model parameters since $\Phi$ and $\Psi$ are not inverse-square in our theory but a repeat analysis using the solutions of (5) and (6) would yield constraints at the same level.

\section{Acknowledgments}

We would like to thank Phil Bull and Claudia Maraston for several enlightening correspondences.

\section{References}

1. A. Joyce, B. Jain, J. Khoury and M. Trodden (2014) arXiv:1407.0059 [astro-ph.C0].

2. A. Vainshtein, Phys.Lett. B39 (1972) 393.

3. G. Dvali, G. Gabadadze and M. Porrati, Phys.Lett. B485 (2000) 208, arXiv:hep-th/0005016 [hep-th].

4. C. de Rham, G. Gabadadze and A. J. Tolley, Phys.Rev.Lett. 106 (2011) 231101, arXiv:1011.1232 [hep-th]

5. E. Babichev, C. Deffayet and R. Ziour, JHEP 0905 (2009) 098, arXiv:0901.0393 [hep-th]

6. S. Hassan and R. A. Rosen, JHEP 1202 (2012) 126, arXiv:1109.3515 [hep-th]

7. C. Deffayet, G. Esposito-Farese and A. Vikman, Phys.Rev. D79 (2009) 084003, arXiv:0901.1314 [hep-th]

8. R. Kimura, T. Kobayashi and K. Yamamoto, Phys.Rev. D85 (2012) 024023, arXiv:1111.6749 [astro-ph.C0]

9. K. Koyama, G. Niz and G. Tasinato, Phys.Rev. D88 (2013) 021502, arXiv:1305.0279 [hep-th] 
10. A. Nicolis, R. Rattazzi and E. Trincherini, Phys.Rev. D79 (2009) 064036, arXiv:0811.2197 [hep-th].

11. R. P. Woodard, Lect.Notes Phys. 720 (2007) 403, arXiv:astro-ph/0601672 [astro-ph].

12. N. Afshordi, G. Geshnizjani and J. Khoury, JCAP 0908 (2009) 030, arXiv:0812.2244 [astro-ph].

13. G. W. Horndeski, Int.J.Theor.Phys. 10 (1974) 363.

14. A. Barreira, B. Li, C. M. Baugh and S. Pascoli, Phys.Rev. D86 (2012) 124016, arXiv:1208.0600 [astro-ph.CO]

15. A. Barreira, B. Li, C. Baugh and S. Pascoli (2013) arXiv:1308.3699 [astro-ph.C0].

16. M. Zumalacrregui and J. Garca-Bellido, Phys.Rev. D89 (2014) 064046, arXiv:1308.4685 [gr-qc].

17. J. Gleyzes, D. Langlois, F. Piazza and F. Vernizzi (2014) arXiv:1404.6495 [hep-th].

18. J. Gleyzes, D. Langlois, F. Piazza and F. Vernizzi (2014) arXiv:1408.1952 [astro-ph.CO].

19. X. Gao, Phys.Rev. D90 (2014) 081501, arXiv:1406.0822 [gr-qc]

20. X. Gao, Phys.Rev. D90 (2014) 104033, arXiv:1409.6708 [gr-qc]

21. K. Koyama and J. Sakstein (2015) arXiv:1502.06872 [astro-ph.CO]

22. T. Kobayashi, Y. Watanabe and D. Yamauchi (2014) arXiv:1411.4130 [gr-qc]

23. A.-C. Davis, E. A. Lim, J. Sakstein and D. Shaw, Phys.Rev. D85 (2012) 123006, arXiv:1102.5278 [astro-ph.CO]

24. B. Paxton et al., Astrophys. J. Suppl. 192 (2011) 3, arXiv:1009.1622 [astro-ph.SR].

25. B. Paxton, M. Cantiello, P. Arras, L. Bildsten, E. F. Brown et al. (2013) arXiv:1301.0319 [astro-ph.SR]

26. J. F. Navarro, C. S. Frenk and S. D. White, Astrophys.J. 462 (1996) 563, arXiv:astro-ph/9508025 [astro-ph].

27. A. F. Neto, L. Gao, P. Bett, S. Cole, J. F. Navarro et al., Mon.Not.Roy.Astron.Soc. 381 (2007) 1450, arXiv:0706.2919 [astro-ph]

28. SDSS Collaboration Collaboration (X. Xue et al.), Astrophys.J. 684 (2008) 1143, arXiv:0801.1232 [astro-ph].

29. R. Swaters, R. Sanders and S. McGaugh, Astrophys.J. 718 (2010) 380, arXiv: 1005.5456 [astro-ph.CO]

30. J. Mastache, J. L. Cervantes-Cota and A. de la Macorra, Phys.Rev. D87 (2013) 063001, arXiv: 1212.5167 [astro-ph.GA].

31. V. Vikram, J. Sakstein, C. Davis and A. Neil (2014) arXiv:1407.6044 [astro-ph.CO].

32. J. Moffat and V. Toth, Phys.Rev. D91 (2015) 043004, arXiv:1411.6701 [astro-ph.GA].

33. F. Schmidt, Phys.Rev. D81 (2010) 103002, arXiv:1003.0409 [astro-ph.C0].

34. J. Schwab, A. S. Bolton and S. A. Rappaport, Astrophys.J. 708 (2010) 750, arXiv:0907.4992 [astro-ph.CO] 